\title{
Combined use of SSRIs and NSAIDs increases the risk of gastrointestinal adverse effects
}

\author{
Jeroen C. F. de Jong, Paul B. van den Berg, Hilde Tobi \& Lolkje T. W. de Jong-van den Berg \\ Department of Social Pharmacy and Pharmacoepidemiology, Groningen University Institute for Drug Exploration, (GUIDE), University Centre for \\ Pharmacy, Groningen, The Netherlands
}

\begin{abstract}
Aims To investigate the relationship between the use of antidepressants with or without NSAIDs and the risk of gastrointestinal side-effects.

Methods This was a population-based cohort study. Medication data of 180000 patients from 16 pharmacies in The Netherlands were studied. The subjects were a group of 15445 new users of antidepressants with or without NSAIDs. A review of patient profiles for cases of gastrointestinal adverse effects caused by first time use of antidepressants with or without NSAIDs was carried out. The number of first prescriptions for peptic ulcer drugs (Anatomical Therapeutic Chemical classification A02B) in the period from day 2 after starting antidepressants with or without NSAIDs until 10 days after the last dose was the main outcome measure.

Results In the reference group of 619 new users of nonselective antidepressants (TCAs), the incidence of first prescriptions for peptic ulcer drugs was 0.051 (95\% confidence interval $0.021,0.105)$. The use of SSRIs $(n=1181)$ caused a slightly higher incidence rate ratio (IRR) of $1.2(0.5,2.8)$. The combined use of SSRIs and NSAIDs $(n=86)$ increased the IRR to $12.4(3.2,48.0)$. In contrast, the combination of nonselective antidepressants and NSAIDs $(n=74)$ increased the IRR to 2.5 $(0.3,20.3)$.

Conclusions SSRIs increase the risk of gastrointestinal adverse effects in first time users as compared with nonselective antidepressants. The combined use of SSRIs and NSAIDs strongly increases the risk of gastrointestinal adverse effects and should be avoided. The combination of nonselective antidepressants and NSAIDs does not have this effect.
\end{abstract}

Keywords: adverse effects, antidepressants, cohort study, gastrointestinal drugs, nonsteroidal anti-inflammatory drugs, selective serotonin reuptake inhibitors

\section{Introduction}

Selective serotonin reuptake inhibitors (SSRIs) such as the frequently prescribed antidepressants fluoxetine and paroxetine are thought to increase the risk of gastrointestinal bleeding by blocking the reuptake of serotonin in the thrombocytes $[1,2]$. For nonselective tricyclic antidepressants (TCAs), clearly defined gastrointestinal adverse effects have not been described.

Correspondence: Professor Dr L. T. W. de Jong-van den Berg, Department of Social Pharmacy and Epidemiology, Groningen, University Institute for Drug Exploration (GUIDE), A. Deusinglaan I, 9713 AV Groningen, The Netherlands. Tel: + 3 I 5036 33330; Fax: +3I 5036 32772; E-mail: I.t.w.de.jong-van.den.berg @farm.rug.nl

Received 22 July 2002, accepted 14 October 2002.
Nonsteroidal anti-inflammatory drugs (NSAIDs) are widely prescribed for various indications. Apart from their anti-inflammatory, analgesic and antipyretic actions, they exert serious adverse effects on the thrombocytes, the kidneys and the gastrointestinal tract. Subjective gastropathy is the most frequently reported adverse effect, occurring in up to half the number of all NSAID users [3]. The prevalence of ulcers is high [4].

Until recently there were no epidemiological reports about the possible gastrointestinal adverse effects caused by the combined use of SSRIs and NSAIDs. In 1999 De Abajo et al. [5] performed a case-control study and reported a relative risk of the combination of 15.6, compared with 3.7 for NSAIDs and 3.0 for SSRIs alone. In a concurrent editorial, Li Wan Po [6] concluded that the combination leads to an increased risk far beyond a simple 
additive effect, and that further studies using alternative study designs are necessary to confirm these results.

In Dutch pharmacies the prescription medication of all patients is carefully monitored, and warnings about possible adverse effects, contraindications, and interactions are consequently generated. After the publication of De Abajo et al. [5], the interaction of NSAIDs with SSRIs was incorporated in the monitoring systems of the Dutch community pharmacies, but there was no information available about the consequences of this interaction. Therefore in 2000 one of the monitoring programs stopped reporting the possible risks of the combination.

To assess the magnitude and the implications of the interaction between SSRIs and NSAIDs in everyday practice, we carried out a population-based cohort study to investigate the relationship between the use of antidepressant drugs (SSRIs and TCAs) with or without NSAIDs, and the risk of gastrointestinal adverse effects.

\section{Methods}

Design

The study was a population-based cohort study to investigate the association between the use of antidepressant drugs with or without NSAIDs and the risk of gastrointestinal side-effects.

If drug A or a certain combination of drugs has a sideeffect and drug $B$ is prescribed to counteract this specific side-effect, drug B is the proxy drug for this side-effect. Once its use as a proxy has been validated, it can provide quantitative information about the risk of the side-effect [7].

In this study peptic ulcer drugs $\left(\mathrm{H}_{2}\right.$-receptor blocking agents, proton pump inhibitors, prostaglandins) of the Anatomical Therapeutic Chemical (ATC) classification A02B [8] were used as proxy drugs for gastrointestinal adverse effects. In 1990 Leufkens [9] validated this proxy for gastrointestinal adverse effects.

\section{Setting}

The study was performed using the InterAction DataBase (IADB) in Groningen. It contains the demographic and the prescription data of about 180000 patients from 16 community (nonhospital) pharmacies in the northeastern part of The Netherlands. This IADB and its use in study designs has been described previously [10].

\section{Subjects}

A selection was made of all patients in the IADB who were born before 1 January 1981. Excluded were all users of drugs of the ATC groups B01A (thrombocyte aggregation inhibitors) and $\mathrm{H} 02$ (systemic corticosteroids). For each patient the IADB contains demographic data (age, gender) and pharmacy data (generic name, strength, dosage, route of administration, day of issue and prescription length in days). The IADB contains no information about the indications for the prescription of the drugs.

We selected all 1961 patients who were new users of antidepressant drugs [SSRIs (ATC $=$ N06AB) and TCAs $($ ATC $=$ N06AA) $]$ with or without NSAIDs $(\mathrm{ATC}=\mathrm{M} 01 \mathrm{~A})$ in the study period between 1 July 1999 and 30 June 2000. By new users we mean that they had no prescription for antidepressants, for NSAIDs or for peptic ulcer drugs in the 6 months before the study period. The data of one patient were incomplete; this patient was excluded. The final study group consisted of 1960 patients.

\section{Exposure definition}

The exposure time was defined as the number of patient years that antidepressant drugs with or without NSAIDs were taken before either a first prescription for gastric protection with a peptic ulcer drug or the end of the study period. The exposure time was calculated as the total number of doses divided by 366 days and the number of defined daily doses (DDD) of the specific drug. Patients who received gastroprotective drugs such as omeprazole or misoprostol as prophylaxis were excluded from the study.

\section{Outcome definition}

The outcome was defined as the number of first prescriptions for peptic ulcer drugs in the period from day 2 after starting the use of antidepressants with or without NSAIDs until 10 days after the last dose.

\section{Analysis}

The group of 619 new users of nonselective antidepressants was chosen as a reference group. Incidence rates (IR) were calculated by dividing the number of first prescriptions for gastric protection by the total number of patient years at risk. The incidence rate ratios (IRR) were calculated as $I R R=I R_{\text {index group }} / \mathrm{IR}_{\text {reference group }}$ All results were calculated with their corresponding 95\% confidence intervals $(95 \% \mathrm{CI})$, using CIA computer software [11].

\section{Results}

The main characteristics of the study population are given in Table 1. The mean ages in the index groups and the reference group were 41.0-44.6 [standard deviation [(SD]) 14.3-15.7] years and $45.9 \pm 15.8$ years, respectively. 
Table 1 Characteristics of the study population.

\begin{tabular}{lcccc}
\hline & $\begin{array}{c}\text { TCA (reference) group } \\
(\mathrm{n}=619)\end{array}$ & $\begin{array}{c}\text { SSRI group } \\
(\mathrm{n}=1181)\end{array}$ & $\begin{array}{c}\text { SSRI }+ \text { NSAID group } \\
(\mathrm{n}=86)\end{array}$ & $\begin{array}{c}\text { TCA + NSAID group } \\
(\mathrm{n}=74)\end{array}$ \\
\hline Gender & & & & \\
$\quad$ Male & $209(33.8 \%)$ & $435(36.8 \%)$ & $56(34.9 \%)$ & $28(37.8 \%)$ \\
$\quad$ Female & $410(66.2 \%)$ & $746(63.2 \%)$ & $22-91$ & $46(62.2 \%)$ \\
Age range (years) & $18-89$ & $18-91$ & 43.2 & $19-91$ \\
Mean age & 45.9 & 41.0 & 14.6 & 44.6 \\
SD mean age & 15.8 & 14.3 & - & 14.5 \\
Incomplete data & 1 & - & - \\
\hline
\end{tabular}

Table 2 Incidence rates (IR) and incidence rate ratios (IRR) in the study groups.

\begin{tabular}{|c|c|c|c|c|c|c|c|}
\hline Group & $\begin{array}{c}\text { Number of } \\
\text { patients }\end{array}$ & $\begin{array}{l}\text { Exposure } \\
\text { number of } \\
\text { patients } \\
\times \text { years }\end{array}$ & $\begin{array}{c}\text { Number } \\
\text { of pepti } \\
\text { culcer drugs }\end{array}$ & $\begin{array}{l}\text { Incidence } \\
\text { rate (IR) }\end{array}$ & $95 \% C I$ & $\begin{array}{c}\text { Incidence } \\
\text { rate ratio } \\
(I R R)\end{array}$ & $95 \% C I$ \\
\hline Reference (TCA) & 619 & 137 & 7 & 0.051 & $0.021,0.105$ & 1 & - \\
\hline SSRI & 1181 & 383 & 23 & 0.060 & $0.038,0.090$ & 1.2 & $0.5,2.8$ \\
\hline NSAID + SSRI & 86 & 4.73 & 3 & 0.634 & $0.131,1.854$ & 12.4 & $3.2,48.0$ \\
\hline NSAID + TCA & 74 & 7.87 & 1 & 0.127 & $0.003,0.708$ & 2.5 & $0.3,20.3$ \\
\hline
\end{tabular}

In the TCA (reference) group (Table 2) there were seven first prescriptions for a peptic ulcer drug, giving an incidence rate of 0.051 [95\% confidence interval [(CI]) 0.021, 0.105].

The use of SSRIs was associated with an incidence rate (IR) of peptic ulcer drug use of $0.060(0.038,0.090)$; the incidence rate ratio (IRR) was $1.2(0.5,2.8)$, indicating a slightly higher, but not significant risk of requiring a peptic ulcer drug in SSRI users compared with TCA users.

The combined use of SSRIs and NSAIDs, however, strongly increased the incidence rate of first prescriptions for peptic ulcer drugs to $0.634(0.131,1.854)$ and the incidence rate ratio to $12.4(3.2,48.0)$, indicating a significantly higher risk of requiring a peptic ulcer drug in users of the combination of SSRIs and NSAIDs compared with users of TCAs alone or SSRIs alone.

In contrast, the combination of TCAs with NSAIDs had an incidence rate of $0.127(0.003,0.708)$, and an incidence rate ratio of $2.5(0.3,20.3)$, indicating that the use of the combination of TCAs and NSAIDs does not result in a significant increase in the risk of requiring a peptic ulcer drug.

\section{Discussion}

Our results confirm earlier reports [5] that the use of SSRIs in combination with NSAIDs does increase the risk of gastrointestinal adverse effects: about 10 times higher than for SSRIs alone and about four times higher than the reported risk [5] for NSAIDs alone. Nonselective antidepressants in combination with NSAIDs do not have this effect.

In order to be able to link the use of antidepressants with or without NSAIDs to an increased risk of gastrointestinal adverse effects we have imposed rigid conditions on the searches in the IADB. A valid relationship was only acknowledged if a prescription for peptic ulcer medication, as a proxy for gastrointestinal adverse effects, was given from day 2 after starting antidepressants with or without NSAIDs until day 10 after the theoretical end of use. Several guidelines $[12,13]$ recommend that patients at higher risk of developing NSAID-induced gastropathy should receive concomitant therapy with a gastroprotective drug, such as omeprazole $[14,15]$ or misoprostol $[16,17]$. We have not included patients with such automatic, concomitant protection by starting our analysis on day 2 after starting the antidepressant medication with or without NSAIDs. In this way we have increased the likelihood that a prescription for peptic ulcer medication is directly caused by gastrointestinal adverse effects of antidepressants with or without NSAIDs. However, it will also underscore the causal relationship. As we have no knowledge about the indications for the prescribed drugs, there is no absolute certainty about the causal relationship, nor of the severity 
of the adverse effects. The rigid conditions that have been imposed on the searches are all the more necessary because the threshold for the use of gastroprotective drugs has been substantially lowered since 1990, the date of the validation of this proxy marker. $\mathrm{H}_{2}$-receptor antagonists are now even available as OTC drugs. Previously these drugs were mostly reserved for patients with proven ulcers; nowadays irregular dyspeptic or reflux symptoms are also regarded as an indication for occasional use. However, we regard the clear sequence of the use of a gastroprotective drug after the initiation of SSRIs with or without NSAIDs as a strong indication of the causal relationship.

SSRIs alone increase the risk of gastrointestinal adverse effects [5]. They block the reuptake of serotonin by the thrombocytes, resulting in an impairment of the haemostatic function [3, 4]. In their case-control study De Abajo et al. [5] calculated an odds ratio of 3.0. In a reaction to this publication, Williams et al. reported an odds ratio of 1.6 [18]. In our study the incidence rate ratio is 1.2 compared with the use of nonselective antidepressants. A possible explanation of this lower incidence rate ratio is that patients who are at risk of gastrointestinal adverse effects, and therefore concomitantly use protective peptic ulcer drugs, are not included. De Abajo et al. [5] and Williams et al. [18] report small increases in the risk for gastrointestinal adverse effects of these nonselective antidepressants. Table 3 lists the results of the three studies.

In this cohort study the combined use of SSRIs and NSAIDs resulted in a large increase in the incidence rate from 0.051 to 0.634 and in the incidence rate ratio to 12.4 as compared with the TCA (reference) group. However, this number may be an underestimation. NSAIDs are not only prescribed and reimbursed, but they are also sold without a physician's prescription as an OTC drug, probably in substantial numbers [19]. Because it is likely that patients in the index group and in the reference group take such OTC drugs, the measured incidence rate ratio may be influenced, although we cannot measure the actual magnitude of the influence. Moreover, not all gastric ulcers induced by NSAIDs are treated [20, 21].
De Abajo et al. [5], in their case-control study on clinically diagnosed upper gastrointestinal haemorrhages, report an odds ratio of 15.6 for the same combination. However, for the combination of TCAs with NSAIDs we found a relatively small increase in the incidence rate of adverse effects from 0.051 for users of TCAs to 0.127 for users of the combination of TCAs with NSAIDs; the incidence rate ratio of 2.5 is in the same range as the risk for NSAIDs alone [5]. This difference in IRR implies that the use of peptic ulcer drugs is not caused by the depressive condition alone, but in substantial part by using NSAIDs together with SSRIs or TCAs.

The large increase in risk for patients using an SSRI and an NSAID concomitantly should have consequences for everyday practice. The medication monitoring systems in Dutch community pharmacies should routinely generate warning signals about gastrointestinal adverse effects whenever such combinations are prescribed, and these signals should be taken seriously. The same applies to Dutch practitioners as prescribers of drugs, some of them even connected to the monitoring system of pharmacies. There are several options to decrease the risk of gastrointestinal adverse effects. NSAIDs could be used for a shorter duration or in a lower dosage, or could be replaced by paracetamol (acetaminophen). In addition, a NSAID that is more COX-2 selective could be chosen, because from a theoretical point it seems likely that COX-2-selective NSAIDs cause fewer gastrointestinal side-effects [22, 23]. SSRIs could be replaced by TCAs. So far there are no reports about the adverse effects caused by the combination of SSRIs with COX-2 selective NSAIDs.

In cases where the combination of SSRIs and NSAIDs cannot be avoided, standard protection with peptic ulcer drugs could be considered to prevent gastrointestinal complaints. The concomitant use of $\mathrm{H}_{2}$-receptor antagonists in high dosage [24], proton pump inhibitors [14, 15], and prostaglandin analogues [15] can be considered. For patients at high risk, such as those over 60 years of age [23] and with a history of previous NSAID-induced gastrointestinal symptoms [2], standard comedication of peptic ulcer drugs can prevent potential serious gas-

\begin{tabular}{lccc}
\hline Group & $\begin{array}{c}\text { IRR } \\
\text { (this study) }\end{array}$ & $\begin{array}{c}\text { Adjusted OR } \\
\text { (De Abajo et al. [5]) }\end{array}$ & $\begin{array}{c}\text { OR } \\
\text { (Williams et al. [18]) }\end{array}$ \\
\hline NSAID & - & $3.7(3.2,4.4)$ & - \\
SSRI & $1.2(0.5,2.8)$ & $3.0(2.1,4.4)$ & $1.6(1.4,1.7)$ \\
TCA & 1 & $1.4(1.1,1.9)$. & $1.7(1.6,1.9)$ \\
NSAID + SSRI & $12.4(3.2,48.0)$ & $15.6(6.6,36.6)$ & - \\
NSAID + TCA & $2.5(0.3,20.3)$ & - & - \\
\hline
\end{tabular}

Table 3 Comparison of IRRs with (adjusted) ORs from the studies of De Abajo et al. [5] and Williams et al. [18] (95\% CI in brackets). 
trointestinal adverse effects. It will, however, not always prevent the development of NSAID-induced gastric ulcers.

In conclusion, our results confirm that particularly the use of SSRIs in combination with NSAIDs increases the risk of gastrointestinal adverse effects, a 10 times higher risk than for SSRIs alone. Nonselective antidepressants do not have this effect. The warning signals generated by the medication monitoring systems should be taken seriously, and efforts should be made to avoid the combination of SSRIs and NSAIDs.

JCFdJ initiated and executed the study, participated in designing, analysis and writing of the study. PBvdB participated in designing and reporting the study, HT participated in designing, analysis and reporting of the study. LTWdJ-vdB participated in designing, analysis and writing of the study, and is the guarantor.

\section{References}

1 Cooper TA, Valcour VG, Gibbons RB, O’Brien-Falls K. Spontaneous ecchymoses due to paroxetine administration. Am J Med 1998; 104: 197-198.

2 Alderman CP, Moritz CK, Ben-Tovim DI. Abnormal platelet aggregation associated with fluoxetine therapy. Ann Pharmacother 1992; 26: 1517-1519.

3 Wolfe MM, Lichtenstein DR, Singh G. Gastrointestinal toxicity of nonsteroidal antiinflammatory drugs. N Engl J Med 1999; 340: 1888-1899.

4 Singh G. Recent considerations in nonsteroidal antiinflammatory drug gastropathy. Am J Med 1998; 105 (1B): 31S-38S.

5 De Abajo FJ, García Rodríguez LA, Montero D. Association between selective serotonin reuptake inhibitors and upper gastrointestinal bleeding: population based case-control study. Br Med J 1999; 319: 1106-1109.

6 Li Wan Po A. Antidepressants and upper gastrointestinal bleeding: new results suggest a link. Br Med J 1999; 319: 1081-1082.

7 Petri H, De Vet HCW, Naus J, Urquhart J. Prescription sequence analysis: a new and fast method for assessing certain adverse reactions of prescription drugs in large populations. Statis Med 1988; 7: 1171-1175.

8 Anatomical Therapentical Chemical (ATC) Classification Index Including Daily Defined Dosages (Ddds) for Plain Substances. Oslo: World Health Organisation Collaborating Centre for Drug Statistics Methodology; 2000.

9 Leufkens HGM. Pharmacy records in pharmacoepidemiology: studies on antiinflammatory and antirheumatic drugs. Thesis. Utrecht, 1990; 137-146.

10 Tobi H, Van den Berg PB, De Jong-van den Berg LTW. The InterAction database: synergy of science and practice in pharmacy. Lect Notes Comput Sci 2000; 1933: 206211.

11 Bryant TN. Confidence Interval Analysis (CIA) Software 2000, Version 2.0.0I. In Statistics With Confidence, 2nd edn, eds Altman DG, Machin D, Bryant TN, Gardner MJ. Bristol: BMJ Books, 2000.

12 American College of Rheumatology Subcommittee on Osteoarthritis Guidelines. Recommendations for the medical management of osteoarthritis of the hip and the knee. 2000 update. Arthritis Rheum 2000; 43: 1905-1915.

13 Rostom A, Wells G, Tugwell P, Welch V, Dube C, McGowan J. Prevention of NSAID-induced gastroduodenal ulcers (Cochrane Review). In The Cochrane Library Issue 2. Oxford: Update Software, 2001.

14 Yeomans ND, Tulassey Z, Juhász L, et al. A comparison of omeprazole with ranitidine for ulcers associated with nonsteroidal antiinflammatory drugs. N Engl J Med 1998; 338: 719-726.

15 Hawkey CJ, Karrasch JA, Szczepanski L, et al. Omeprazole compared with misoprostol for ulcers associated with nonsteroidal antiinflammatory drugs. N Engl J Med 1998; 338: 727-734.

16 Silverstein FE, Graham DY, Senior JR, et al. Misoprostol reduces serious gastrointestinal complications in patients with rheumatoid arthritis receiving nonsteroidal anti-inflammatory drugs: a randomized, double-blind, placebo-controlled trial. Ann Intern Med 1995; 123: 241-249.

17 Plosker GL, Lamb HM. Diclofenac/misoprostol: pharmacoeconomic implications of therapy. Pharmacoeconomics 1999; 16: 85-98.

18 Williams D, Kelly A, Feely J. Coprescription of antiulcer drugs with SSRIs is fairly common. Br Med J 2000; 320: 1405.

19 Dickinson T, Malhi S, Painter S, Pyott J, Sawhney A. Self treatment with non-steroidal drugs may be confounding factor. $\mathrm{Br}$ Med J 2000; 320: 1405.

20 Skander MP, Ryan FP. Non-steroidal anti-inflammatory drugs and pain free peptic ulceration in the elderly. $\mathrm{Br}$ Med J 1988; 297: 833-834.

21 Janssen M, Dijkmans BAC, Lamers CBHW, Zwinderman AH, Vandenbroucke JP. A gastroscopic study of the predictive value of risk factors for non steroidal anti-inflammatory drugassociated ulcer disease in rheumatoid arthritis patients. $\mathrm{Br} J$ Rheumatol 1994; 33: 449-454.

22 Lems WF, Van de Laar MAFJ, Bijlsma JWJ. Veiligheid van specifieke cyclo-oxygenase-2-remmers [Safety of specific cyclo-oxygenase 2 inhibitors]. Ned Tijdschr Geneesk 2001; 145: 1044-1047.

23 Bombardier C, Laine L, Reicin A, et al. Comparison of upper gastrointestinal toxicity of rofecoxib and naproxen in patients with rheumatoid arthritis. N Engl J Med 2000; 343: 15201528 .

24 Agrawal NM, Aziz K. Prevention of gastrointestinal complications associated with nonsteroidal antiinflammatory drugs. J Rheumatol 1998; 25 (S 51): 17-20. 